\title{
When transcription meets recombination: a lesson from the human RECQ protein complexes Yilun $\mathrm{Liu}^{1 *}$ and Joan W Conaway ${ }^{2,3}$
}

\author{
Addresses: ${ }^{1}$ Department of Therapeutic Radiology, Yale University School of Medicine, New Haven, CT 06520-8040, USA; ${ }^{2}$ Stowers Institute for \\ Medical Research, 1000 East $50^{\text {th }}$ Street, Kansas City, MO 64110-2262, USA; ${ }^{3}$ Department of Biochemistry and Molecular Biology, \\ Kansas University Medical Center, 2146 West $39^{\text {th }}$ Street, Kansas City, KS 66160, USA \\ * Corresponding author: Yilun Liu (yilun.liu@yale.edu) \\ FI000 Biology Reports 2009, I:76 (doi:I0.34I0/BI-76)
}

The electronic version of this article is the complete one and can be found at: http://FI000.com/Reports/Biology/content/I/76

\begin{abstract}
Since the cloning of the first human RECQ gene, RECQ I, more than I5 years ago, RECQ helicases have been a major focus in cancer research. Recent studies of human RECQ protein complexes are providing insight into their roles in various DNA metabolic pathways that protect the integrity of our genome.
\end{abstract}

\section{Introduction and context}

The highly conserved RecQ helicase was first identified in Escherichia coli about 25 years ago and was subsequently found to be required for suppressing illegitimate recombination in both E. coli and yeast [1-3]. Ten years later, discovery of the link between the human homolog, BLM, and Bloom syndrome demonstrated the importance of the RecQ family helicases in human health [4]. Bloom syndrome is a devastating genetic disorder that causes cancer predisposition. Cells derived from Bloom syndrome patients show high levels of sister chromatid exchange resulting from DNA recombination and genome rearrangements reminiscent of those found in lower organisms [5].

Importantly, BLM is not the only RECQ homolog to be identified in humans. During evolution, the RecQ gene has been amplified and has diverged from a single copy in bacteria to five RECQ homologs in humans, namely, RECQ1, BLM, WRN, RECQ4, and RECQ5. Importantly, although there are multiple REC helicases in humans, they are not redundant, as mutations in different RECQ helicases are responsible for distinct clinical diseases [6]. Bloom syndrome, Werner syndrome, and RothmundThomson syndrome have been linked to defects in BLM, WRN, and RECQ4, respectively. In the past decade, much research has been dedicated to uncovering molecular functions of RECQ family helicases linked with different clinical disorders.

\section{Major recent advances}

Among the five human RECQ helicases, BLM has been the most extensively studied. BLM is found in a complex with topoisomerase III $\alpha$ (TOPOIII $\alpha$ ) and the RecQmediated genome instability (RMI) proteins RMI1 and RMI2. Cumulative data indicate that BLM makes use of four possible mechanisms to repair DNA breaks by both promoting and suppressing homologous recombination (Figure 1) [7]. First, BLM, together with TOPOIII $\alpha$ and RMI1/2, dissolves double Holliday junction intermediates into non-crossover products [8]. Second, BLM may contribute to the synthesis-dependent single-stranded annealing pathway, leading exclusively to products without sister chromatid exchange [9]. Most recently, BLM has been implicated in two opposite roles: disassembling the RAD51 filament from the break site to prevent homologous recombination; and facilitating end resection to promote homologous recombination [10-12].

While it is clear that BLM is an essential player in safeguarding the progression and influencing the outcome of homologous recombination events, how these pro- and anti-recombinational roles of BLM are coordinated during 
Figure I. Potential roles of BLM and RECQ5 in sister chromatid exchange regulation

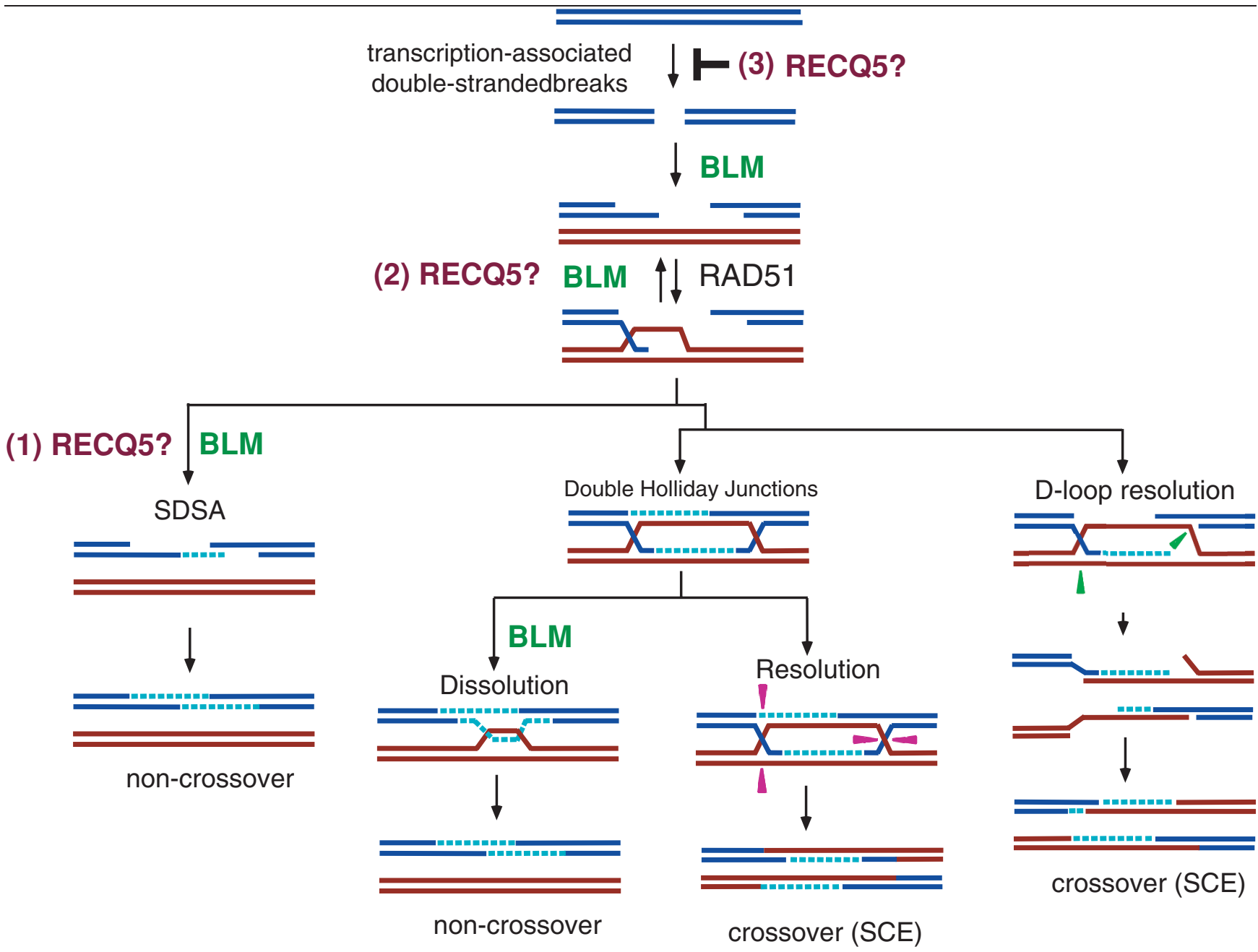

A recombination event is initiated by a DNA break and leads to crossover or non-crossover products. First, the DNA ends are resected to produce single-stranded DNA, which in a reaction catalyzed by RAD5I recombinase can then invade the homologous duplex DNA to form a recombination intermediate, or D-loop structure. After homologous recombination is initiated by RAD5I at one end of the broken DNA, the second DNA end may be captured by the unpaired strand of the homologous DNA. The resulting recombination intermediate can proceed to form double Holliday junctions (HJs), and any remaining gaps may be filled by DNA synthesis. HJs can be cleaved by HJ resolvase (center right) or dissociated by the BLM-dependent pathway (center left). In mitotic cells, it is argued that the majority of homologous recombination is completed without the formation of HJs. Instead, most homologous recombination events are completed by synthesis-dependent strand annealing (SDSA) to generate non-crossover products that result from dissociation of the D-loop after DNA synthesis (left). The newly synthesized DNA is available to re-anneal with the second end of the same DNA molecule at the break site. DNA synthesis continues to fill the gap and restore the integrity of the DNA without crossover. Alternatively, D-loop structure can be cleaved to generate sister chromatid exchange (SCE) products (right). Possible points of RECQ5 action discussed in the text are indicated by (I), (2), and (3).

homologous recombination is still a puzzle. Further complicating the story, it had been thought that elevated levels of sister chromatid exchange accompanied by chromosome rearrangement are a hallmark of Bloom syndrome cells and a key feature in the clinical diagnosis of Bloom syndrome, however, recent studies have shown that mouse embryonic stem cells defective in RECQ5 exhibit a phenotype similar to that of Bloom syndrome cells [13]. Furthermore, even though RECQ5 mutations are not linked to Bloom syndrome or any other clinical disease in humans, RECQ5 deletion in mice results in a significant increase in the development of solid tumors in various organs such as the lung, liver, and breast [14]. Notably, the recq5-/- blm-/- double mutant exhibited an even greater frequency of sister chromatid exchange than either of the single mutants, suggesting that these two proteins contribute to regulation of sister chromatid exchange via different mechanisms [13]. 
While RECQ5 does not dissolve double Holliday junctions [15], it is possible that it may function to promote synthesis-dependent single-strand annealing (Figure 1, mechanism 1). Alternatively, it has been shown that RECQ5 has an antagonistic role in preventing RAD51 filament formation (Figure 1, mechanism 2) [14]. However, both of these RAD51-dependent mechanisms are redundant to BLM function and are not likely to represent the primary role of RECQ5 in suppressing sister chromatid exchanges. Indeed, a recent report demonstrated an additive sensitivity to camptothecin treatment in recq5-rad51 double mutants [16], arguing for a role of RECQ5 in a RAD51-independent pathway.

A recent surprise in the field came with the purification from human cells of a multiprotein complex containing the RECQ5 helicase. Unlike BLM, which forms a stable complex with TOPOIII $\alpha$ and RMI1/2 to function as a 'dissolvasome' in homologous recombination [7], RECQ5 primarily interacts with RNA polymerase II, the enzyme responsible for messenger RNA synthesis in eukaryotes (Figure 2) [17,18]. Importantly, this interaction is specific to RECQ5 but not to other RECQ helicases [17]. Further biochemical analysis revealed that RECQ5 efficiently suppresses RNA polymerase IIdependent transcription via direct protein-protein interaction [19]. Together, these data suggest a unique role of RECQ5 in transcription.

\section{Future directions}

The biochemical activities of the BLM-TOPOIII $\alpha$ complex are clearly consistent with a role in homologous recombination pathways leading exclusively to formation of non-sister chromatid exchange products (Figure 1). On the other hand, many questions remain regarding the physiological connection between the RECQ5-RNA polymerase II interaction and sister chromatid exchange. Nonetheless, the potential link between transcription

Figure 2. Distinct BLM and RECQ5 core protein complexes in human cells

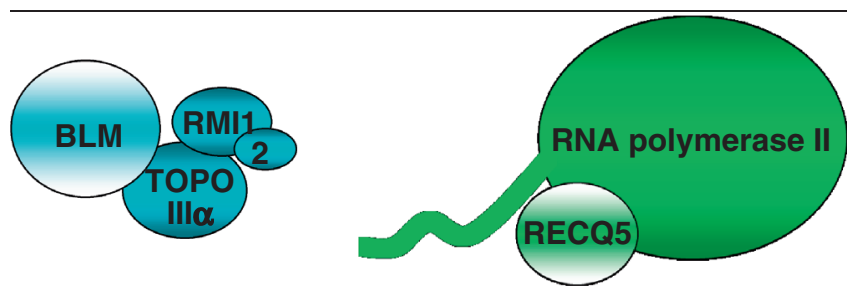

Schematic diagrams of the BLM-TOPOIII $\alpha-R M I I / 2$ complex (left) and the RECQ5-RNA polymerase II complex (right) purified from human cell extracts. RMI, RecQ-mediated genome instability; TOPOIII $\alpha$, topoisomerase III $\alpha$. and recombination may not be unexpected. While transcription is essential for cells to function and grow, the process itself can generate undesirable DNA lesions and result in genome instability [20]. Indeed, mutations in RNA processing factors, such as ASF/SF2 and THO/TREX, result in double-stranded break formation and transcription-associated recombination $[21,22]$. It is very reasonable, therefore, to expect that RECQ5 could have a unique function in preventing DNA break formation during transcription (Figure 1, mechanism 3). According to this hypothesis, transcription-dependent double-stranded breaks would be generated in the absence of RECQ5 and subsequently repaired by homologous recombination, leading to an increase in sister chromatid exchange. It is worth noting that transcription-associated recombination was also observed in yeast [20], yet Sgs1, the only RecQ homolog in yeast, has not been implicated in this process. Further, Sgs1 is not known to interact with RNA polymerase II. Thus, it is possible that a role for RECQlike helicases in transcription-associated recombination has been acquired during the course of evolution and is unique to higher eukaryotes.

Given that the key to solving the puzzle regarding RECQ5 and BLM may lie within the distinct protein complexes they form in cells, the identification of other RECQ helicase complexes may provide important clues to the unique functions of the remaining RECQ helicases in DNA metabolism. Such discoveries would allow us to start to understand how the human RECQ helicases associate with different clinical syndromes that have specific cancer spectra.

\section{Abbreviations}

RMI, RecQ-mediated genome instability; TOPOIII $\alpha$, topoisomerase III .

\section{Competing interests}

The authors declare that they have no competing interests.

\section{Acknowledgements}

Work in YL's laboratory is supported by grants from the National Cancer Institute (5RO1 CA130910) and the Breast Cancer Alliance. Work in JWC's laboratory is supported by a grant from the National Institute of General Medical Sciences (5R37 GM041628) and by the Stowers Institute for Medical Research.

\section{References}

I. Nakayama H, Nakayama K, Nakayama R, Irino N, Nakayama Y, Hanawalt PC: Isolation and genetic characterization of a thymineless death-resistant mutant of Escherichia coli KI2: identification of a new mutation (recQI) that blocks the 
RecF recombination pathway. Mol Gen Genet 1984, I95: 474-80.

2. Hanada K, Ukita T, Kohno Y, Saito K, Kato J, Ikeda H: RecQ DNA helicase is a suppressor of illegitimate recombination in Escherichia coli. Proc Natl Acad Sci U S A 1997, 94:3860-5.

3. Watt PM, Hickson ID, Borts RH, Louis EJ: SGSI, a homologue of the Bloom's and Werner's syndrome genes, is required for maintenance of genome stability in Saccharomyces cerevisiae. Genetics 1996, 144:935-45.

4. Ellis NA, Groden J, Ye TZ, Straughen J, Lennon DJ, Ciocci S, Proytcheva M, German J: The Bloom's syndrome gene product is homologous to RecQ helicases. Cell 1995, 83:655-66.

5. German J: Bloom syndrome: a mendelian prototype of somatic mutational disease. Medicine 1993, 72:393-406.

6. Hanada K, Hickson ID: Molecular genetics of RecQ helicase disorders. Cell Mol Life Sci 2007, 64:2306-22.

7. Liu Y, West SC: More complexity to the Bloom's syndrome complex. Genes Dev 2008, 22:2737-42.

8. Wu L, Hickson ID: The Bloom's syndrome helicase suppresses crossing over during homologous recombination. Nature 2003, 426:870-4.

FI000 Factor 6.4 Must Read

Evaluated by Lucio Comai I5 Jan 2004, Steven Brill 2I Jan 2004

9. Bachrati $\mathrm{CZ}$, Borts RH, Hickson ID: Mobile D-loops are a preferred substrate for the Bloom's syndrome helicase. Nucleic Acids Res 2008, 34:2269-79.

10. Bugreev DV, Yu X, Egelman EH, Mazin AV: Novel pro- and antirecombination activities of the Bloom's syndrome helicase. Genes Dev 2007, 21:3085-94.

II. Gravel S, Chapman JR, Magill C, Jackson SP: DNA helicases Sgs I and BLM promote DNA double-strand break resection. Genes Dev 2008, 22:2767-72.

FI000 Factor 3.0 Recommended

Evaluated by Nick Rhind 18 Feb 2009

12. Nimonkar AV, Ozsoy AZ, Genschel J, Modrich P, Kowalczykowski SC: Human exonuclease I and BLM helicase interact to resect DNA and initiate DNA repair. Proc Natl Acad Sci U S A 2008, I05:16906-II.
13. Hu Y, Lu X, Barnes E, Yan M, Lou H, Luo G: RecQL5 and BIm RecQ helicases have non-redundant roles in suppressing crossovers. Mol Cell Biol 2005, 25:343 I-42.

14. Hu Y, Raynard S, Sehorn MG, Lu X, Bussen W, Zheng L, Stark JM, Barnes EL, Chi P, Janscak P, Jasin M, Vogel H, Sung P, Luo G: RECQL5/RecqI5 helicase regulates homologous recombination and suppresses tumor formation via disruption of Rad5 I presynaptic filaments. Genes Dev 2007, 2 I:3073-84.

15. Wu L, Lung C, Ralf C, Bernstein DA, Garcia PL, Bohr VA, Vindigni A, Janscak P, Keck JL, Hickson ID: The HRDC domain of BLM is required for the dissolution of double Holliday junctions. EMBO J 2005, 24:2679-87.

16. Hu Y, Lu X, Zhou G, Barnes EL, Luo G: Recql5 plays an important role in DNA replication and cell survival after camptothecin treatment. Mol Biol Cell 2009, 20: I |4-23.

17. Aygun O, Svejstrup J, Liu Y: A RECQ5-RNA polymerase II association identified by targeted proteomic analysis of human chromatin. Proc Natl Acad Sci U S A 2008, 105:8580-4.

18. Izumikawa K, Yanagida M, Hayano T, Tachikawa H, Komatsu W, Shimamoto A, Futami K, Furuichi Y, Shinkawa T, Yamauchi Y, Isobe T, Takahashi N: Association of human DNA helicase RecQ5beta with RNA polymerase II and its possible role in transcription. Biochem J 2008, 413:505-16.

19. Ozan A, Xu X, Liu Y, Takahashi H, Kong SE, Conaway RC, Conaway JW, Svejstrup JQ: Direct inhibition of RNA polymerase II transcription by RECQL5. J Biol Chem 2009, 284: $23197-203$.

20. Aguilera A: The connection between transcription and genomic instability. EMBO J 2002, 2 I:195-201.

21. Li X, Manley JL: Inactivation of the SR protein splicing factor ASF/SF2 results in genomic instability. Cell 2005, I 22:365-78.

FI000 Factor 6.0 Must Read Evaluated by Sui Huang 20 Dec 2005

22. Wellinger RE, Prado F, Aguilera A: Replication fork progression is impaired by transcription in hyperrecombinant yeast cells lacking a functional THO complex. Mol Cell Biol 2006, 26: 3327-34. 